\title{
The Measurement of Barium Titanate's Curie Temperature Based on Hysteresis Loop
}

\author{
Chenlei Zhao ${ }^{1, \text { a }}$ \\ ${ }^{1}$ School of physics, Nanjing University, Nanjing 210093, China; \\ azhaochenlei502@sina.com
}

Keywords: Curie temperature, hysteresis loop, ferroelectric, dielectric constant.

\begin{abstract}
In an applied electric field, the relationship between the ferroelectric's intensity of polarization and the electric field intensity is not linear but a hysteretic loop which is called the ferroelectric hysteresis loop. When the temperature of the ferroelectric is heated to the transition temperature (the Curie temperature), its dielectric properties experience a mutation, changing from the ferroelectric phase into the paraelectric phase. This article discusses a way to measure the ferroelectric's Curie temperature by changing the ferroelectric's temperature and observing the change in the ferroelectric hysteresis loop.
\end{abstract}

\section{Introduction}

Ferroelectric, an important kind of electronic materials, has been developed for thousand series since its first discovery in the 1920s. Ferroelectric has many unique properties such as piezoelectric effect, thermo electric effect, electro-optic effect, acousto-optic effect, etc. These unique properties make its prosperous application in electronic technology, laser technology, and computing technology. [1] BaTiO3 has a typical ABO3 structure, and since its ferroelectricity first discovered in 1942, it has been widely used in military, aerospace and household electrical appliances. Many research shows the measurement of BaTiO3 Curie temperature is important, while it has not been well established due to the limitation of experimental platform. [2, 3, 4]

Without an applied electric field, the centers of the positive and negative charges in the crystal are not coincident, which appears as electric dipole moments. The crystals that have spontaneous polarization and whose direction of the polarization can change according to the direction of the applied electric field are called ferroelectrics. The ferroelectric hysteresis loop is the ferroelectrics' unique property and by observing its change with temperature, we can find the ferroelectric's Curie temperature. [5] Based on some self-assembled laboratory equipment, I successfully measured the ferroelectric's Curie temperature and analyzed the cause of the change in the ferroelectric hysteresis loop.

\section{Method}

In some temperature ranges, the centers of the positive and negative charges in the crystal are not coincident, which appears as electric dipole moments. The directions of some electric dipole moments are coherent, which forms the so-called ferroelectric domain.

The basic property of ferroelectrics is that its spontaneous polarization's direction turns according to the applied electric field. Figure.1 shows the ferroelectric hysteresis loop of typical ferroelectrics. While there is no applied electric field, the ferroelectric domains' distribution is random so that the crystal shows electric neutrality in total. When the crystal is in an applied electric field, the ferroelectric domains that have the same direction as the electric field increase and those that have the opposite direction decrease. Thus, the intensity of polarization (P) increases with the electric field intensity (E), like the OA part of Figure.1. With the enlargement of the electric field intensity, all the ferroelectric domains turn to the same direction, like a single ferroelectric domain, at this time the intensity of polarization is saturated. If the electric field intensity continues to increase, the polarization intensity increases linearly with the electric field intensity, shown as the $\mathrm{C}$ part of the 
Figure.1. Prolong the linear part to $\mathrm{E}=0$ and the intercept of the $\mathrm{P}$ axis is called the spontaneous polarization intensity (Ps). If the electric field decreases from $\mathrm{C}$ part, the polarization intensity also decreases. At $\mathrm{E}=0$ there is still remanent polarization (Pr). This is because when the electric field intensity decreases, some ferroelectric domains diverge from the original direction because of the tension in the crystal. But when E comes to 0, most ferroelectric domains still remain the original direction so that macroscopically, there is remanent polarization. Keep decreasing the electric field intensity until it reverses to -Ec, the remanent polarization disappears. Ec is called the coercive electric field intensity.

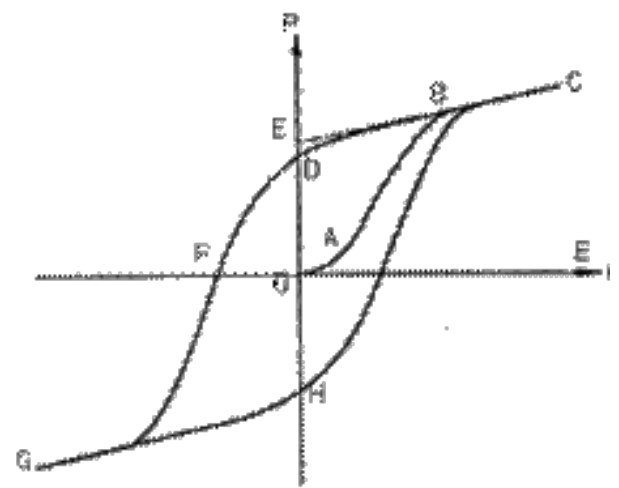

Figure. 1 ferroelectric hysteresis loop

Since the ferroelectric's dielectric constant, coefficient of elasticity and spontaneous polarization are influenced by temperature, it is of great interests to investigate there effects. [6] When temperature rises, the thermal motion of every structure cell is faster. So the coherence of electric dipole moments' direction is breached more or less. The electric dipoles that get some freedom overcome the tendency to be chaotic caused by the thermal motion and turn to the same direction as the applied electric field. So the dielectric constant increases with temperature. When temperature rises to a specific point (Tc), all the ferroelectric domains disintegrate so the ferroelectricity disappears and so does the ferroelectric hysteresis loop. This temperature is called the Curie temperature. Obviously, the dielectric constant reaches its top at the Curie temperature. If temperature keeps rising, there is no more electric dipole released from the ferroelectric domain. And its distribution is more chaotic because of thermal motion. So the dielectric constant decreases while temperature keeps rising. The turn of the dielectric constant is so quick that it appears as a mutation. And macroscopically it appears as mutation in the remanent polarization (Pr).

During the experiment, the $\mathrm{BaTiO}_{3}$ sample was put into a heating furnace and connected the sample with the TF_DH1 measuring instrument for ferroelectric hysteresis loop with wires. Also, a thermocouple is applied to detect the temperature in the furnace. After connecting the instruments, voltage was applied to the sample. And on the screen of the measuring instrument a complete ferroelectric hysteresis loop can be observed to test the integrity of the sample. After that, the heating furnace was turned on, sample was heated gradually, the change in the ferroelectric hysteresis loop is observed and the remanent polarization and the coercive electric field intensity at each temperature are recorded. When the temperature reached the estimated Curie temperature, the heating was slowed down and the change in the ferroelectric hysteresis loop was recorded carefully until the temperature reached and passed the Curie temperature. After the temperature passed the Curie temperature for a while and the ferroelectric hysteresis loop stopped changing, the heating furnace was turned off and sample went through a natural cooling process. Then the ferroelectric hysteresis loop was observed and recorded again. At last the two groups of data were reorganized into a chart and were calculated with computer. Eventually the Curie temperature was get.

\section{Results}

In the experiment, I noticed that the width of the ferroelectric hysteresis loop kept shrinking. However, this is a gradual process and I didn't observe a mutation. Further conclusion needs quantitative analysis. 
The change of the remanent polarization and the coercive electric field intensity with temperature is presented as follows:
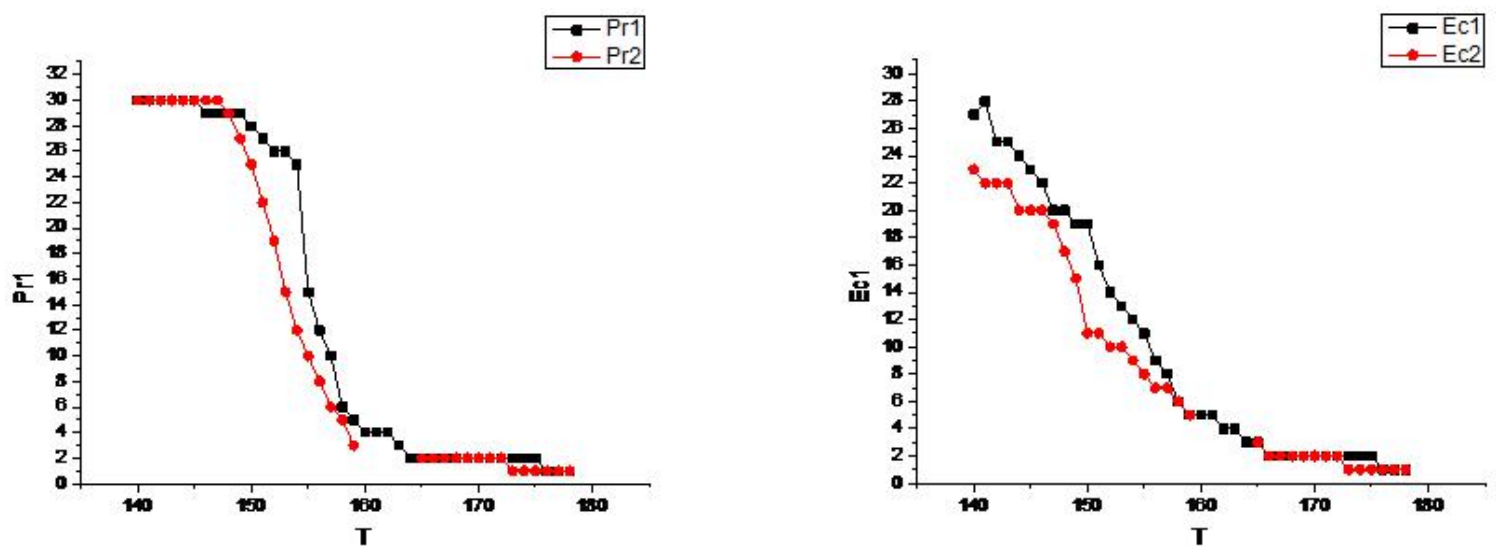

Figure. 2 (a),(b) change of the Pr and the Ec with temperature

Figure. 2 illustrates the change of the remanent polarization and the coercive electric field intensity with temperature. The black line represents the temperature's rising process and the red line represents the temperature's declining process. Although there wasn't a mutation in the shape of the ferroelectric hysteresis loop, we can see from the data, especially the data of the remanent polarization that there does exist a mutation in the process. In order to find out the mutation temperature, I polynomial fit the data by the software OriginPro and made a derivative process based on it to find out the mutation temperature by finding out the extremum of the derivative.

The result of the derivative is presented as follows:
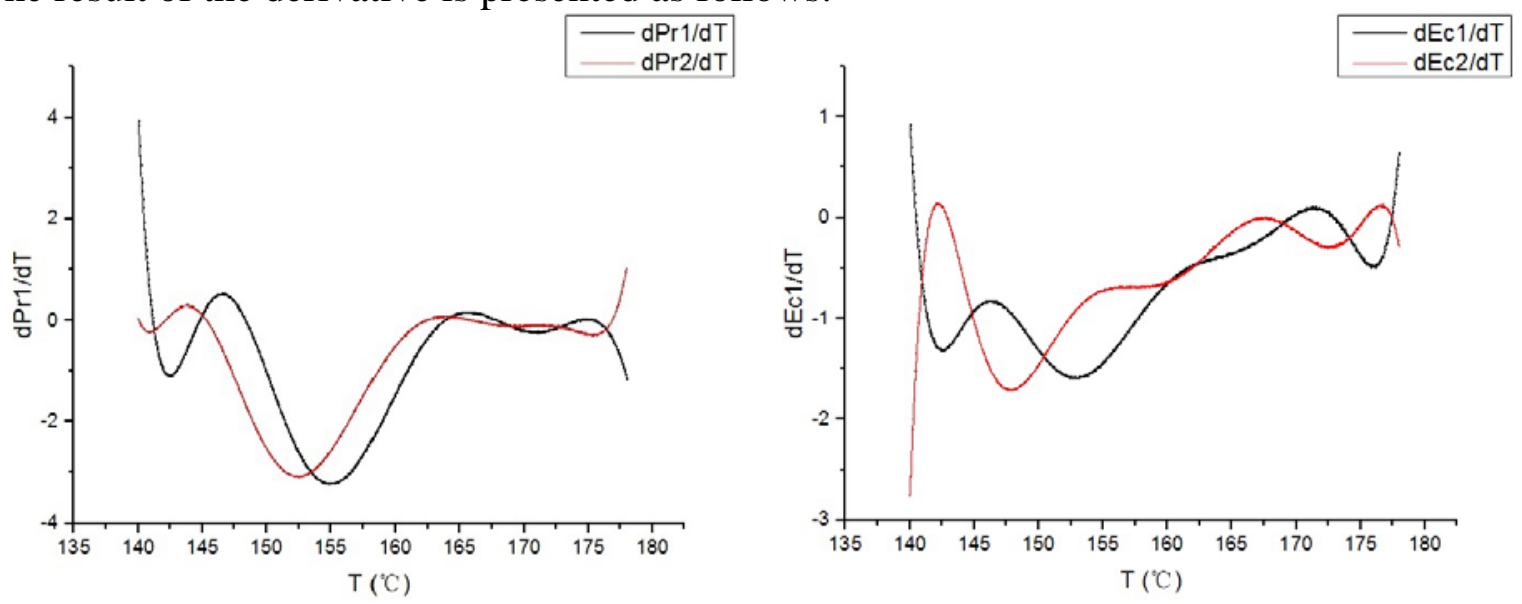

Figure. 3 (a), (b) derivative of the change of the Pr and the Ec with temperature

According to the calculation, in the temperature's rising process, the extremum of the remanent polarization's derivative is at $T_{P 1}=154.87^{\circ} \mathrm{C}$ and the extremum of the coercive electric field intensity is at $T_{E 1}=152.51^{\circ} \mathrm{C}$. In the temperature's declining process, the extremum of the remanent polarization's derivative is at $T_{P 2}=152.32^{\circ} \mathrm{C}$ and the extremum of the coercive electric field intensity is at $T_{E 2}=147.80^{\circ} \mathrm{C}$. Considering that the mutation in the remanent polarization is more obvious, I took the mutation temperature of the remanent polarization as the Curie temperature. Calculate the average of the data of the rising process and the declining process to get the final result:

The Curie temperature is:

$$
T_{C}=\frac{T_{P 1}+T_{P 2}}{2}=153.60^{\circ} \mathrm{C}
$$




\section{Summary}

This experiment is based on the theory of ferroelectric's phase change and the ferroelectric hysteresis loop. The Curie temperature of the BaTiO3 was measured using a laboratory developed equipment. Also, I noticed that during the experiment, the change in the shape of the ferroelectric hysteresis loop was less than I had thought but there does exist a mutation during the change of the temperature. It's just that the mutation appears in the remanent polarization rather than the shape. However, the temperature shown by the thermocouple is not very accurate because it's not connected to the sample directly considering the possibility of leakage of electricity. To measure the Curie temperature more accurately, there must be a new way to detect the temperature. The Curie temperature is an important physical quantity of a material. The applied range of a material is limited greatly by the Curie temperature. So the measurement of the Curie temperature is very important. This article presented a simple way to do so, but I'm sure there will be more accurate way to measure it in the future and it will help make new breakthroughs in the application of many new materials.

\section{References}

[1]He Yansong. A Comprehensive Study on Mechanical-Electric Nonlinear Coupling Behavior of Ferroelectric Materials [D]. Chongqing University, 2003.

[2]Zhang Y, Deng X, Han L, et al. Effect factors on the domain switching current in BaTiO_3 ceramics with colossal dielectric constant[J]. Journal of Functional Materials, 2012, 7: 025.

[3]Frey M H, Payne D A. Grain-size effect on structure and phase transformations for barium titanate[J]. Physical Review B, 1996, 54(5): 3158

[4]Liu Jia, Yang Renbo, Deng Xiangyun, et al. Temperature and applied field effect on ferroelectric properties of nanocrystalline BaTiO3 ceramics [J]. Journal of Functional Materials, 2013, 43(24): 3364-3367.

[5] Wu Jing, Li Wenfang, Huang Wenbo. Micromechanics Analysis of the Influence of Temperature on the Process of Ferroelectric Polarization Reversal [j]. Chinese Journal of Solid Mechanics, 2009, 30(4): 341-345.

[6] Franco Jona, G Shirane. Ferroelectric Crystals [M]. Dover Publications, 1993. 108- 225. 